\title{
LA SUSTITUIBILIDAD DE LA MANO DE OBRA CALIFICADA EN LAS EMPRESAS INDUSTRIALES
}

\author{
George Psacharopoulos * \\ London School of Economics \\ Universidad de Londres
}

\section{INTRODUCCIÓN}

ANTES DE que alguien se embarque en un ejercicio de planificación de mano de obra, tiene que dar respuesta a una serie de cuestiones preliminares. En primer lugar tendrá que escoger la unidad de análisis. ¿Debería tratar de predecir el número de personas con distinta calificación educativa o el de personas dentro de los distintos grupos ocupacionales? ¿Sería más estable la estructura ocupacional o la educativa para propósitos de predicción? O tal vez, ¿debería proceder en dos etapas al predecir primero la estructura ocupacional y traducirla luego a la estructura educativa? Y en caso de que decida hacer esto último, ¿cómo se llevará a cabo esta traducción?

Suponiendo que este problema se haya resuelto, la siguiente pregunta se refiere a la facilidad de sustitución entre diferentes clases de fuerza de trabajo. En realidad, ¿importaría si para el año previsto la economía tuviera más ingenieros que el número estrictamente "requerido" para alcanzar los objetivos de producción que establece el plan nacional? Porque en el caso de una economía rígida donde los ingenieros sobrantes no pudieran absorberse fácilmente en otros empleos y permanecieran sin empleo, el producto nacional sería inferior al máximo, debido a una mala planificación de la mano de obra y al desperdicio de recursos resultante. Sin embargo, en el caso de una economía flexible, los ingenieros sobrantes podrían absorberse fácilmente y así se daría menos interés a la predicción de "requerimientos de mano de obra" exactos.

En la cuestión acerca de la facilidad de sustitución entre insumos de producción diferentes, está implícita la de la productividad marginal de las clases de trabajo que uno trata de predecir. ¿Es esta productividad marginal igual a los salarios observados en el mercado? ¿Cómo podrá cambiar la productividad marginal de un cierto tipo de trabajo después de que las proporciones de las personas con cualidades diferentes en la economía hagan otro tanto? En una economía rígida, un tipo de trabajo dado se podría sustituir por otros factores de producción sólo con el cre-

* Un informe más detallado acerca del Proyecto LSE de Mano de Obra Industrial, aparece en P. R. G. Layard, I. D. Sargan, M. E. Ager y D. J. Jones, Qualified Manpower and Economic Performance, Londres, Penguin Press, de próxima publicación. Los puntos de vista expresados en este trabajo son completamente personales y los autores del libro no necesariamente participan de ellos. 
cimiento sustancial de los costos unitarios. Lo contrario sería verdad en el caso de una economía caracterizada por la facilidad de sustitución, y donde diversas proporciones entre factores serían compatibles con costos unitarios más o menos constantes.

Una cuestión que surge a menudo en la planificación de la mano de obra es el análisis de tipo costo-beneficio. Dadas las productividades marginales de las diferentes clases de trabajo y los costos de producirlas, ¿es redituable producir más de un cierto tipo o de otro? Y si lo es producir cierto tipo de mano de obra, ¿cuánto más debe ser ésta en términos absolutos? Aún más, las medidas de redituabilidad, tal como se calcularon con base en la presente información de corte transversal, ¿serán válidas para una proyección?, o ¿las nuevas proporciones de personas en la fuerza de trabajo invalidarán las antiguas medidas de redituabilidad?

Debiera ser obvio que la pregunta crucial en este contexto es la de la facilidad de sustitución entre clases de trabajo diferentes. La razón es que la respuesta a esta pregunta dictará automáticamente la metodología a usarse en el ejercicio de la planeación de la mano de obra. Existen por lo menos dos métodos distintos y comparativos en la planificación de la mano de obra: el enfoque de "requerimientos de mano de obra" y el de la "tasa de beneficio". De acuerdo con el primero, importa realmente predecir el número exacto de mano de obra de distinta calificación para el año meta. Sin el número requerido de personal, no podrá alcanzarse la meta de producción nacional. Las posibilidades de sustitución en la economía son muy limitadas o prácticamente nulas. La mano de obra requerida debe producirse a cualquier costo. En contraste, el enfoque de la tasa de beneficio sostiene que las facilidades de sustitución en la economía son ilimitadas. La predicción del número exacto de personas con niveles educativos diferentes para el año meta no importa realmente, pues cualquiera que sea la combinación que surja de personas con diferentes calificaciones, la economía las absorberá todas con facilidad. Lo que importa en este enfoque es el costo de producir una persona calificada de un tipo dado relativo a los beneficios que esta persona proporcionaría a la economía como un todo. Sin embargo, este enfoque requiere estimar la redituabilidad de la inversión en educación, a fin de concluir en qué dirección debiera cambiar el producto educativo. Los números absolutos del año meta no importan realmente.

Desde luego, los dos enfoques presentados representan casos extremos. La realidad yace en algún lugar dentro del continuo definido por la completa flexibilidad y por los casos totalmente rígidos. Sin embargo, para seguir una metodología particular en la planificación de la mano de obra, nos gustaría, para propósitos prácticos, saber si estamos más cerca de un extremo del continuo o del otro. ${ }^{1}$

\section{EL PROYECTO L.S.E. DE MANO DE OBRA INDUSTRIAL}

En muchas partes del mundo la planificación de la mano de obra se ha llevado a cabo basada en supuestos más que en evidencia empírica,

1 En M. Blaug, "Approaches to Educational Planning", The Economic Journal, junio de 1967, se encontrará un examen de los supuestos usados en los distintos enfoques. 
respecto de las cuestiones cruciales mencionadas antes. Por ejemplo, el Proyecto Regional Mediterráneo de la ocDE, procedió con base en el supuesto de que la demanda de los distintos tipos de trabajo está determinada tecnológicamente y de este modo se usó el enfoque de requerimientos de mano de obra. ${ }^{2}$ El Proyecto LSE de Mano de Obra Industrial se diseñó para proporcionar respuestas empíricas a algunas de las preguntas que se mencionan anteriormente, a las cuales se encaran los planificadores educativos. En particular, interesa la facilidad de sustitución y la predicción de diferentes tipos de trabajo que utiliza una empresa.

El tipo de mano de obra que se estudió fue el de científicos e ingenieros calificados en la industria eléctrica de Gran Bretaña. La unidad de análisis fueron fábricas con 200 o más trabajadores en 1967. El número total de personal asalariado estudiado fue de aproximadamente 26000 , que pertenecían a 68 establecimientos. De la muestra se obtuvieron dos tipos de información: primero, los detalles sobre cada empleado calificado y, segundo, los relativos al establecimiento en que trabajaban. La información sobre el trabajador comprendió: edad, nivel educativo, posición ocupacional, función y sueldo. La referente al establecimiento incluyó: productos bruto y neto, naturaleza del producto, tamaño de la casa matriz, artículos y servicios comprados, número total de empleados, costo del trabajo y capital fijo y de trabajo.

Para propósitos analíticos se distinguieron cuatro niveles de calificación educativa. El primer nivel abarcó empleados de tiempo completo con grado educativo alto, como licenciaturas, maestrías y doctorados. Señalaremos este grupo de empleados con el nombre de "graduados". El otro grupo de empleados, al que llamaremos "profesionales", abarca a aquellos que obtuvieron su calificación técnica después de cierto número de años en la profesión y los que pasaron un examen especial administrado por algún instituto profesional (por ejemplo, del Instituto de Ingenieros Eléctricos). La tercera calificación la referimos como cNS, y consiste de empleados con cuatro o cinco años de estudios parciales durante el empleo, después de la edad escolar obligatoria. La última categoría consiste de empleados calificados como cNo, que son aquellos con dos o tres años de estudios de medio tiempo y empleados después de la escuela secundaria obligatoria. ${ }^{3}$

El cuadro 1 muestra la distribución de los empleados calificados en los 68 establecimientos de la muestra. Los profesionales graduados son alrededor de $1 \%$ de la fuerza de trabajo de cada fábrica, mientras que el porciento correspondiente a los CNS y CNO es el doble. Lo que resulta interesante para nuestros propósitos es la dispersión entre fábricas del porciento de empleados con distintas calificaciones.

La columna 3 del cuadro 1 muestra grandes variaciones en la proporción de fuerza de trabajo calificada de los establecimientos, y esta observación es particularmente importante en el caso del extremo superior de nuestro espectro de calificación, o sea los graduados con educación superior.

2 En R. Hollister, A Technical Evaluation of the First Stage of the Mediterraenan Regional Project, París: OCDE, 1966, puede verse una valoración técnica de este importante ejercicio de planificación de la mano de obra.

3 Las iniciales corresponden como sigue: Certificado Nacional Superior (CNs) y Certificado Nacional Ordinario ( $\mathrm{CNO}$ ). Ver M. Blaug, An Introduction to the Economics of Education, Londres, Penguin, 1970, pp. 327-29, que contiene un glosario de los términos educativos británicos. 


\section{Cuadro 1}

MEDiAS Y DESVIACIONES ESTÁNDAR DEL PORCIENTO DE EMPLEADOS POR GRUPO EDUCATIVO

\begin{tabular}{lcc}
\hline $\begin{array}{l}\text { Grupo educativo } \\
\text { (1) }\end{array}$ & $\begin{array}{c}\text { Media } \\
(2)\end{array}$ & $\begin{array}{c}\text { Desviaoín } \\
\text { es tándar } \\
\text { (3) }\end{array}$ \\
\hline 1. Graduados & 1.16 & 1.58 \\
2. Profesionales & 0.98 & 0.72 \\
3. Certificado Nacional Superior & 2.25 & 1.66 \\
(CNS) & 2.03 & 1.23 \\
4. Certificado Nacional Ordinario & (CNO) & 25 \\
\hline
\end{tabular}

Notas: Los porcientos que aparecen en la columna (2) se refieren al total de empleados del establecimiento.

Renglón 1. Grado de educación superior que se obtuvo en estudios de tiempo completo.

Renglón 2. Calificación obtenida al pasar exámenes profesionales después de estudiar medio tiempo y cierta experiencia en una profesión dada.

Renglón 3. Calificación obtenida después de cinco años de estudio de medio tiempo y empleo después de la escuela secundaria obligatoria.

Rerglón 4. Calificación obtenida después de dos o tres años de estudios de medio tiempo y empleo después de la escuela secundaria obligatoria.

La cuestión inmediata que surge es por qué la proporción de personas con diferentes calificaciones varía tanto entre las distintas fábricas. ¿Esto se debe a la diferente combinación de productos, al método de producción, a los sueldos relativos del mercado o a otros posibles factores? La respuesta a esta pregunta es muy importante, ya que de existir una relación unívoca entre las características de los establecimientos y la proporción de fuerza de trabajo empleada, hay posibilidad de predecir "los requerimientos de mano de obra" para llevar a cabo los objetivos de producción. En este caso, la demanda de determinada clase de mano de obra podría estar determinada más o menos tecnológicamente. Por una parte, si no existe relación alguna entre la proporción de personas y las características de las empresas, concluiríamos que las proporciones exactas de mano de obra realmente no importan. Pero si por alguna razón las diferentes empresas se las ingenian para acomodar distintas proporciones de calificación en la composición de su fuerza de trabajo, sin reducir la eficiencia, se concluiría que no es necesario predecir los requerimientos exactos de mano de obra. Para explicarlo de otra manera, si el producto se puede generar eficientemente por medio de muy diversas combinaciones de insumos, todo intento de planificar en forma exacta las proporciones de mano de obra podría no tener sentido.

En el contexto particular de esta muestra, nos gustaría saber si la variación de la proporción de empleados calificados se debe al hecho de que algunas empresas producen maquinaria eléctrica pesada, tales como turbogeneradores, mientras que otras producen componentes electrónicos como transistores, condensadores y resistencias. ¿O se debe tal vez a que algunas empresas son ineficientes y no maximizan sus ganancias? $¿ \mathrm{O}$ se debe esta variación a otros factores que no podemos cuantificar aunque sepamos que están presentes, y que influyen de manera impredecible en la decisión de la empresa al emplear mano de obra? 


\section{FUNCIONES PRODUCCIÓN}

Casi todas las preguntas que nos hemos formulado pueden contestarse en el contexto de la función producción. Al unir la relación entre el producto y los distintos insumos de la empresa, puede estimarse la elasticidad de sustitución entre los distintos tipos de mano de obra, encontrarse el producto marginal de cada factor de la producción, saberse cómo cambiarán los productos marginales después de un cambio en la proporción de factores empleados, y verse si la naturaleza del producto de cada empresa tiene algo qué ver con la proporción de mano de obra de las distintas calificaciones que emplea. Por lo tanto, nuestra primera tarea fue la de ajustar a la información muestral funciones producción del tipo general siguiente:

$$
Y_{i}=f\left(D_{i}, K_{i}, N_{i}\right)
$$

donde $Y_{i}$ es el producto neto o bruto del establecimiento $i$,

$D$ es un grupo de variables artificiales (dummy) que corresponden al producto particular del establecimiento,

$K$ es una medida del insumo total de capital (a menudo se divide en dos variables separadas, "capital fijo" y "capital de trabajo"), y

$N$ es un grupo de variables que representan los insumos de mano de obra con calificaciones específicas.

Los tipos de producto que se distinguieron (variables $D$ ) fueron maquinaria eléctrica, cables, aparatos telefónicos, electrónica, aparatos domésticos y otros. Los diferentes tipos de insumos de mano de obra que se distinguieron (variable $N$ ) fueron graduados, profesionales, CNS, CNO y sub-cNO. (Véase el cuadro 1.)

En relación con la forma matemática de la función, se ajustaron dos conjuntos de formas: las funciones Cobb-Douglas y las funciones tipo CES. ${ }^{4}$ Las razones por las cuales se ajustaron funciones tipo CES son que la función Cobb-Douglas supone implícitamente que la elasticidad de sustitución entre dos insumos cualesquiera es igual a uno. Luego, tuvimos que ajustar funciones tipo cEs para estimar la elasticidad de sustitución entre distintos tipos de mano de obra, así como entre los diferentes insumos de mano de obra y otros factores de la producción.

Los resultados de las funciones producción al usar la información de la muestra fueron muy decepcionantes. Aun cuando los ajustes de las funciones fueron buenos como se infiere de la variación explicada de la variable dependiente, los elevados errores estándar de las estimaciones no permitieron hacer una inferencia adecuada acerca de la contribución de determinados insumos a la producción ni, en especial, sobre la elasticidad de sustitución entre distintos tipos de mano de obra. En ningún caso se pudo rechazar la hipótesis de que la elasticidad de sustitución entre distintos tipos de insumos es infinita. Esto equivale a decir que todos los insumos de nuestra muestra parecían ser sustitutos perfectos.

4 Función de elasticidades de sustitución constantes. 
Ya que las funciones producción más complicadas del tipo ces no proporcionaron ningún resultado mejor que el de las funciones producción Cobb-Douglas tradicionales, se presentan aquí únicamente los resultados de esta última. El cuadro 2 ofrece algunos resultados típicos de los intentos de estimar las funciones producción. Por ejemplo, de acuerdo con la especificación de la función en la columna 2 de este cuadro, sólo el capital físico y la mano de obra no calificada contribuyen significativamente al producto neto. $\mathrm{Ni}$ el tipo de producto (a juzgar por los coeficientes de las seis variables artificiales) ni ninguno de los cuatro insumos de mano de obra calificada parecen tener relación con el producto del establecimiento. Aún más, el coeficiente del insumo de "graduados" tiene un signo negativo inesperado.

La desagregación del insumo total de capital en capital fijo y de trabajo muestra que el componente de capital fijo es el que se relaciona más estrechamente con el producto (columna 3 ). Sustituyendo en la regresión el producto neto por el producto bruto e incluyendo como insumo los productos que se compraron, no resultó estadísticamente significativo el insumo de mano de obra calificada (columna 4). La agregación del insumo de fuerza de trabajo en una categoría que comprende la subCNO y la CNO y más, mejora marginalmente la relación, ya que la variable artificial que denota el producto tipo "otros" llega a ser estadísticamente significativa (columna 5). Se obtiene el mismo resultado estableciendo la línea divisoria de la agregación de fuerza de trabajo entre el grupo sub-CNS y el cNS y más (columna 6). Una vez más, el capital físico y el insumo de trabajo de calificaciones educativas bajas son los que más se relacionan estadísticamente con el producto. El cuadro 3 muestra otro intento de estimación de las funciones producción dentro de ciertos productos tipo y para cierto proceso de producción. Como en el caso anterior, el resultado fue el mismo. Los que más se relacionan con el producto son los insumos comprados, el capital y la fuerza de trabajo de nivel educativo bajo.

¿Por qué las funciones producción dieron resultados tan deleznables en el sentido de que en ningún caso los coeficientes de regresión de la mano de obra calificada fueron estadísticamente distintos de cero? ¿Se debería a que la información no era correcta? ¿Sería que las empresas no maximizaron las ganancias? ¿O es que nuestra estimación incorpora importantes sesgos estadísticos y de agregación? La sospecha de la calidad de la información se puede desechar rápidamente. La información ha sido reunida meticulosamente por encuestadores calificados y su veracidad fue cotejada cuidadosamente. La segunda posibilidad también puede rechazarse ya que, como se muestra más adelante, las empresas de nuestra muestra parecen combinar con eficiencia los recursos disponibles. Sin embargo, el chivo expiatorio deben ser los sesgos estadísticos que se colaron en nuestros cálculos.

En primer lugar tenemos problemas de agregación. Aunque estamos tratando con la industria de ingeniería eléctrica, el producto de esta industria dista de ser homogéneo. La desagregación por seis tipos de producto o por proceso de producción no parece ser suficiente para separar las distintas funciones producción que supuestamente se aplican a un subconjunto de productos más detallado. Por ejemplo, si un establecimiento produce varios artículos, cada uno con su función producción 
Cuadro 2

Gran Bretaña: Resultado de las funciones producción en la industria ELÉCTRICA-TOTAL DE LOS ESTABLECIMIENTOS

\begin{tabular}{|c|c|c|c|c|c|}
\hline Variable dopendiente & $\begin{array}{l}\text { Produc to } \\
\text { ne to } \\
\text { (2) }\end{array}$ & $\begin{array}{l}\text { Produc to } \\
\text { neto } \\
\text { (3) }\end{array}$ & $\begin{array}{l}\text { Producto } \\
\text { bruto } \\
\text { (4) }\end{array}$ & $\begin{array}{l}\text { Produa to } \\
\text { ne to } \\
\text { (5) }\end{array}$ & $\begin{array}{l}\text { Produc to } \\
\text { ne to } \\
(6)\end{array}$ \\
\hline Termino constanto & -0.018 & -0.014 & 0.025 & -0.023 & -0.020 \\
\hline Maquinaria & $\begin{array}{l}0.000 \\
(0.00)\end{array}$ & $\begin{array}{r}0.000 \\
(0.00)\end{array}$ & $\begin{array}{r}0.000 \\
(0.00)\end{array}$ & $\begin{array}{l}0.000 \\
(0.00)\end{array}$ & $\begin{array}{l}0.000 \\
(0.00)\end{array}$ \\
\hline Cables & $\begin{array}{r}0.122 \\
(0.85)\end{array}$ & $\begin{array}{r}0.120 \\
(0.82)\end{array}$ & $\begin{array}{r}0.019 \\
(0.26)\end{array}$ & $\begin{array}{r}0.155 \\
(1.194)\end{array}$ & $\begin{array}{l}0.163 \\
(1.19)\end{array}$ \\
\hline Telofonos & $\begin{array}{r}0.170 \\
(0.78)\end{array}$ & $\begin{array}{c}0.177 \\
(0.80)\end{array}$ & $\begin{array}{l}0.142 \\
(1.38)\end{array}$ & $\begin{array}{r}0.119 \\
(0.56)\end{array}$ & $\begin{array}{c}0.135 \\
(0.64)\end{array}$ \\
\hline Electróniea & $\begin{array}{r}0.036 \\
(0.38)\end{array}$ & $\begin{array}{r}0.053 \\
(0.54)\end{array}$ & $\begin{array}{c}0.024 \\
(0.53)\end{array}$ & $\begin{array}{l}0.045 \\
(0.47)\end{array}$ & $\begin{array}{l}0.036 \\
(0.37)\end{array}$ \\
\hline Apara tos domés ticos & $\begin{array}{l}-0.174 \\
(1.19)\end{array}$ & $\begin{array}{l}-0.152 \\
(1.02)\end{array}$ & $\begin{array}{r}-0.090 \\
(1.30)\end{array}$ & $\begin{array}{l}-0.117 \\
(0.84)\end{array}$ & $\begin{array}{l}-0.115 \\
(0.83)\end{array}$ \\
\hline Otros & $\begin{array}{r}0.240 \\
(1.81)\end{array}$ & $\begin{array}{r}0.260 \\
(1.94)\end{array}$ & $\begin{array}{c}0.079 \\
(1.29)\end{array}$ & $\begin{array}{r}0.292 \\
(2.56)\end{array}$ & $(2.62)^{0.293}$ \\
\hline Capital total & $\begin{array}{l}0.376^{\frac{2}{2}} \\
(4.53)\end{array}$ & - & $\begin{array}{l}0.121^{\mathrm{a}} \\
(2.35)^{-}\end{array}$ & $\begin{array}{l}0.392^{\mathrm{a}} \\
4.72)^{-1}\end{array}$ & $(4.65)^{0.382^{8 /}}$ \\
\hline Capital fijo & - & $\begin{array}{l}0.244^{\mathrm{g}} \\
(3.21)^{2}\end{array}$ & - & - & - \\
\hline Capital de trabajo & - & $\begin{array}{r}0.127 \\
(1.89)\end{array}$ & - & - & - \\
\hline Artfoul os oomprados & - & - & $(13.34)^{0.617^{\mathrm{a}}}$ & - & - \\
\hline Sub-cNo & $\begin{array}{l}0.512^{a / 2} \\
(6.24)^{-1}\end{array}$ & $\begin{array}{l}0.513^{2} \\
(6.16)\end{array}$ & $\begin{array}{l}0.204^{\frac{2}{3}} \\
(5.09)^{-1}\end{array}$ & $\begin{array}{l}0.498^{2} \\
(6.28)\end{array}$ & - \\
\hline cNo & $\begin{array}{l}-0.099 \\
(1.04)\end{array}$ & $\begin{array}{l}-0.098 \\
(1.00)\end{array}$ & $\begin{array}{l}-0.067 \\
(1.52)\end{array}$ & - & - \\
\hline cNS & $\begin{array}{l}0.136 \\
(1.17)\end{array}$ & $\begin{array}{r}0.149 \\
(1.27)\end{array}$ & $\begin{array}{l}0.073 \\
(1.36)\end{array}$ & - & - \\
\hline Profesionales & $\begin{array}{r}0.058 \\
(1.15)\end{array}$ & $\begin{array}{c}0.058 \\
(1.13)\end{array}$ & $\begin{array}{r}0.034 \\
(1.42)\end{array}$ & - & - \\
\hline Graduados & $\begin{array}{l}-0.024 \\
(0.53)\end{array}$ & $\begin{array}{l}-0.030 \\
(0.65)\end{array}$ & $\begin{array}{c}-0.009 \\
(0.44)\end{array}$ & - & - \\
\hline CNO y mán & - & - & - & $\begin{array}{l}0.054 \\
(0.92)\end{array}$ & - \\
\hline Sub-cNs & - & - & - & - & $\begin{array}{l}0.504^{\mathrm{p}} \\
(6.33)^{\prime}\end{array}$ \\
\hline CNS y máa & - & - & - & - & $\begin{array}{r}0.060 \\
(1.08)\end{array}$ \\
\hline $\mathrm{R}^{2}$ & $0.964^{2 /}$ & $0.963^{3 / 2}$ & $0.992^{\mathrm{a}}$ & $0.963^{\mathrm{g} /}$ & $0.965^{2 / 2 / 2}$ \\
\hline
\end{tabular}

a Indica significación estadística a un nivel de $95 \%$ de probabilidad.

Los números entre paréntesis son coeficientes $t$.

El R ${ }^{2}$ se corrige para los grados de libertad.

propia, no debería extrañar que fuera imposible obtener buenos resultados al intentar estimar funciones producción agregadas. Al interpretar los resultados de este proyecto, hay que considerar si los distintos establecimientos o secciones de ellos están en diferentes funciones producción. Otro problema de naturaleza puramente estadística es que la mano de obra calificada contribuye poco al producto en relación con otros factores de la producción, tales como el capital físico y la mano de obra no calificada. Cuando se combina esto con una varianza alta del error de la regresión, el resultado es que la variable mano de obra calificada no alcanza significación estadística. 


\section{FUNCIONES DEMANDA}

En vista de los resultados anteriores a través del enfoque de funciones de producción, hemos invertido la dirección de la causalidad e intentado estimar las funciones demanda directamente para distintos tipos de mano de obra. En otras palabras, la variable dependiente en este caso es la proporción de empleados calificados en la fuerza de trabajo del esta-

\section{Cuadro 3}

Gran Bretaña: Resultados de las funciones de producción POR TIPO DE PRODUCTO Y PROCESO DE PRODUCCIÓN

\begin{tabular}{|c|c|c|c|c|c|c|}
\hline \multirow[b]{2}{*}{ (1) } & \multicolumn{3}{|c|}{ Tipo de produoto } & \multicolumn{3}{|c|}{ Prooeso de produacion } \\
\hline & $\begin{array}{c}\text { Pesado } \\
\text { (2) }\end{array}$ & $\begin{array}{c}\text { Eleotr6nioa } \\
\text { (3) }\end{array}$ & $\begin{array}{l}\text { Otros } \\
(4)\end{array}$ & $\begin{array}{l}\text { Grupo o } \\
\text { unidad } \\
\text { (5) }\end{array}$ & $\begin{array}{c}\text { Semiespeoia- } \\
\text { lizada } \\
\text { (6) }\end{array}$ & $\begin{array}{c}\text { Proceso } \\
\text { en serie } \\
(7)\end{array}$ \\
\hline Tormino cong tanto & -0.023 & 0.044 & 0.034 & 0.000 & 0.065 & 0.049 \\
\hline Capital fijo & $\begin{array}{l}0.434^{\mathrm{a}} \\
(3.35)^{2}\end{array}$ & $\begin{array}{r}0.090 \\
(1.35)\end{array}$ & $\begin{array}{r}0.110 \\
(1.79)\end{array}$ & $\begin{array}{c}-0.411 \\
(2.33)\end{array}$ & $\begin{array}{r}0.196 \\
(2.22)\end{array}$ & $\begin{array}{l}0.116^{9} \\
(2.30)\end{array}$ \\
\hline Capital de trabajo & $\frac{-0.282^{\mathrm{B}}}{(3.42)}$ & $\begin{array}{r}0.111 \\
(1.55)\end{array}$ & $\begin{array}{l}0.033 \\
(0.67)\end{array}$ & $\begin{array}{c}-0.049 \\
(0.67)\end{array}$ & $\begin{array}{r}0.049 \\
(0.81)\end{array}$ & $\begin{array}{r}0.061 \\
(1.35)\end{array}$ \\
\hline Artfoulos comprados & $\begin{array}{l}0.524^{\mathrm{a}} \\
(4.82)\end{array}$ & $\begin{array}{l}0.495^{\circ} \\
(5.99)\end{array}$ & $\begin{array}{l}0.628^{\mathrm{a}} \\
(9.50)\end{array}$ & $(5.18)^{3.13}$ & $\begin{array}{c}0.552^{\mathrm{g}} \\
(7.19)^{-1}\end{array}$ & $\begin{array}{c}0.640^{\mathrm{a}} \\
(12.89)^{\prime}\end{array}$ \\
\hline$S u b-C N O$ & $\begin{array}{c}0.346 \\
(1.91)\end{array}$ & $\begin{array}{c}0.21)^{2} \\
(2.50)\end{array}$ & $\begin{array}{l}0.201^{8} \\
(3.35)^{-1}\end{array}$ & $\begin{array}{r}0.129 \\
(0.85)\end{array}$ & $\begin{array}{r}0.121 \\
(1.81)\end{array}$ & $(4.20)^{8}$ \\
\hline CNO Y más & $\begin{array}{c}-0.033 \\
(0.31)\end{array}$ & $\begin{array}{r}0.031 \\
(0.50)\end{array}$ & $\begin{array}{c}0.012 \\
(0.25)\end{array}$ & $\begin{array}{r}0.178 \\
(2.30)\end{array}$ & $\begin{array}{r}0.006 \\
(0.09)\end{array}$ & $\begin{array}{c}-0.013 \\
(0.35)\end{array}$ \\
\hline $\mathrm{H}^{2}$ & $0.996^{\mathrm{B}}$ & $0.991^{8}$ & $0.989^{\circ}$ & $0.996^{\mathrm{a}}$ & $0.993^{\mathrm{g}}$ & $0.992^{\mathrm{g}}$ \\
\hline
\end{tabular}

a Indica significación estadística a un nivel de $95 \%$ de probabilidad.

En todas las regresiones, la variable dependiente es el producto bruto.

El $\mathrm{R}^{2}$ se corrige para los grados de libertad.

blecimiento, mientras que la variable independiente se refiere a las distintas características de éste. Las funciones de demandas estimadas fueron de la siguiente forma general:

$$
\left(\frac{N_{e}}{N_{i}}\right)=f\left[D_{i}, Y_{i},\left(\frac{Y}{N}\right)_{i}, R_{i}, S_{i}\right]
$$

donde $\left(\frac{N_{e}}{N_{i}}\right)$ es el porciento de empleados en el establecimiento $i$ con calificación educativa $e$,

$D$ es un grupo de variables artificiales (dummy) que describen los tipos de producto y de proceso de producción,

$Y$ es el producto total neto,

$Y / N$ es el producto por persona, o índice de productividad del trabajo,

$R$ es la tasa de rentabilidad del capital que empleó la empresa, o sea el índice de productividad del capital, y

$S$ es el grupo de variables artificiales que representan el tamaño de la casa matriz. 
Una variable que está notoriamente ausente en el lado derecho de la ecuación que se ha presentado es la de los salarios relativos de la mano de obra de calificaciones diferentes. La razón por la que esta variable no se incluyó en las regresiones es que no muestra demasiada variación en la muestra. Hay que recordar que este es un estudio de corte transversal en un punto del tiempo donde todas las empresas contratan mano de obra en un mercado competitivo.

\section{Cuadro 4}

Resultados DE LA FUNCIÓN DE DEMANDA

\begin{tabular}{|c|c|c|c|c|c|c|}
\hline $\begin{array}{c}\text { Variable dependiente } \\
\text { (1) }\end{array}$ & $\begin{array}{l}\text { Solo } \\
\text { graduados } \\
\text { (2) }\end{array}$ & $\begin{array}{c}\text { CNS } y \\
\text { más } \\
(3)\end{array}$ & $\begin{array}{l}\text { CNS y } \\
\text { más } \\
(4)\end{array}$ & $\begin{array}{c}\text { CNS y } \\
\text { más on } \\
\text { IDE } \\
(5)\end{array}$ & $\begin{array}{c}\text { Ténicos } \\
(6)\end{array}$ & $\begin{array}{c}\text { Téonicos } \\
\text { graduados } \\
\text { (7) }\end{array}$ \\
\hline Término constante & 1.25 & 3.03 & 3.47 & 2.01 & 3.39 & 13.70 \\
\hline Induetria pesada on serio & $\begin{array}{c}0.95 \\
(1.03)\end{array}$ & $\begin{array}{c}0.15 \\
(0.07)\end{array}$ & $\begin{array}{c}0.34 \\
(0.18)\end{array}$ & $\begin{array}{l}0.07 \\
(0.05)\end{array}$ & $\begin{array}{l}-0.39 \\
(0.17)\end{array}$ & $\begin{array}{l}18.29 \\
(1.55)\end{array}$ \\
\hline Indus tria pesada en unidades & $\begin{array}{l}0.46 \\
(0.72)\end{array}$ & $\begin{array}{l}2.18 \\
(1.63)\end{array}$ & $\begin{array}{c}2.31 \\
(1.80)\end{array}$ & $\begin{array}{c}0.83 \\
(0.96)\end{array}$ & $\begin{array}{c}0.06 \\
(0.04)\end{array}$ & $\begin{array}{l}6.45 \\
(0.85)\end{array}$ \\
\hline Industria eloctrónica en serie & $\begin{array}{l}3.00^{\mathrm{a}} \\
(4.15)\end{array}$ & $\begin{array}{c}4.58^{\mathrm{a}} \\
(3.16)\end{array}$ & $\begin{array}{l}4.65^{8} \\
(3.20)\end{array}$ & $\begin{array}{l}2.819 \\
(2.87)\end{array}$ & $(1.90)$ & $\begin{array}{l}25.28^{\circ} \\
(3.51)\end{array}$ \\
\hline Industria electrónica en unidades & $\left(3.52^{\mathrm{a}}\right.$ & $\begin{array}{l}4.03^{2} \\
(4.10)\end{array}$ & $\begin{array}{l}4.05^{\mathrm{a}} \\
(3.99)\end{array}$ & $\begin{array}{l}2.58 \mathrm{~g} \\
(3.67)\end{array}$ & $\left(2.40^{9}\right)$ & $\begin{array}{c}9.81 \\
(1.77)\end{array}$ \\
\hline Otroa productos en serio & $\begin{array}{l}0.06 \\
(0.11)\end{array}$ & $\begin{array}{l}-0.64 \\
(0.58)\end{array}$ & $\begin{array}{l}-0.67 \\
(0.60)\end{array}$ & $\begin{array}{l}-0.43 \\
(0.57)\end{array}$ & $\begin{array}{l}-2.44 \\
(1.76)\end{array}$ & $\begin{array}{l}17.15^{\mathrm{B}} \\
(2.38)\end{array}$ \\
\hline Otros produotos por unidades & $\begin{array}{l}0.00 \\
(0.00)\end{array}$ & $\begin{array}{c}0.00 \\
(0.00)\end{array}$ & $\begin{array}{l}0.00 \\
(0.00)\end{array}$ & $\begin{array}{l}0.00 \\
(0.00)\end{array}$ & $\begin{array}{c}0.00 \\
(0.00)\end{array}$ & $\begin{array}{c}0.00 \\
(0.00)\end{array}$ \\
\hline Producto neto por persona & $-0.47)$ & $\overline{-}$ & $\begin{array}{c}0.13 \\
(0.12)\end{array}$ & $\begin{array}{l}-0.01 \\
(0.01)\end{array}$ & $\begin{array}{c}1.14 \\
(0.92)\end{array}$ & $\begin{array}{l}-3.89 \\
(0.50)\end{array}$ \\
\hline Capital total por persone & - & $\begin{array}{l}0.07 \\
(0.19)\end{array}$ & $\overline{-}$ & - & $\overline{-}$ & $\overline{-}$ \\
\hline Tasa de rentabilidad del capital & $\begin{array}{c}1.03 \\
(0.68)\end{array}$ & $\overline{-}$ & $\begin{array}{l}2.12 \\
(0.70)\end{array}$ & $\begin{array}{c}0.43 \\
(0.21)\end{array}$ & $\begin{array}{l}-2.38 \\
(0.72)\end{array}$ & $\begin{array}{l}15.48 \\
(0.68)\end{array}$ \\
\hline Produotividad total de los factores & $\overrightarrow{-}$ & $\begin{array}{l}0.83 \\
(0.76)\end{array}$ & $\overline{-}$ & 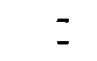 & $\underline{-}$ & $\overline{-}$ \\
\hline Produoto ne to $\left(10^{-3}\right)$ & $\begin{array}{l}0.01 \\
(0.14)\end{array}$ & $\begin{array}{l}-0.15 \\
(1.06)\end{array}$ & $\begin{array}{l}-0.15 \\
(1.10)\end{array}$ & $\begin{array}{l}-0.02 \\
(0.19)\end{array}$ & $\begin{array}{c}-0.15 \\
(0.83)\end{array}$ & $\begin{array}{c}0.51 \\
(0.68)\end{array}$ \\
\hline Katriz pequeña & $\begin{array}{l}-0.84 \\
(1.80)\end{array}$ & $\begin{array}{l}-2.16^{a} \\
(2.34)\end{array}$ & $\begin{array}{l}-2.15 \mathrm{a} / \\
(2.31)\end{array}$ & $\begin{array}{c}-1.50^{a} \\
(2.32)\end{array}$ & $\begin{array}{c}-1.92 \\
(1.53)\end{array}$ & $\begin{array}{c}-1.24 \\
(0.24)\end{array}$ \\
\hline Empresa extranjera grando & $\begin{array}{l}-0.40 \\
(0.66)\end{array}$ & $\begin{array}{l}-0.38 \\
(0.39)\end{array}$ & $\begin{array}{l}-0.27 \\
(0.23)\end{array}$ & $\begin{array}{l}-0.89 \\
(1.09)\end{array}$ & $\begin{array}{l}-2.02 \\
(0.94)\end{array}$ & $\begin{array}{l}3.49 \\
(0.46)\end{array}$ \\
\hline Eropesa britŕnica grande & $\begin{array}{c}0.00 \\
(0.00)\end{array}$ & $\begin{array}{c}0.00 \\
(0.00)\end{array}$ & $\begin{array}{c}0.00 \\
(0.00)\end{array}$ & $\begin{array}{c}0.00 \\
(0.00)\end{array}$ & $\begin{array}{c}0.00 \\
(0.00)\end{array}$ & $\begin{array}{c}0.00 \\
(0.00)\end{array}$ \\
\hline $\mathrm{H}^{2}$ & $0.27^{\mathrm{a} /}$ & $0.32^{\mathrm{a}}$ & $0.33^{\mathrm{a}}$ & $0.28^{\mathrm{a}}$ & $0.38^{\mathrm{g}}$ & 0.19 \\
\hline
\end{tabular}

$a$ indica la significación estadística a un nivel probabilístico de $95 \%$.

La variable dependiente en todas las regresiones excepto la de la columna 7 muestra el porciento de empleados con cierta calificación dada.

La variable dependiente en la columna 7 es el porciento de técnicos con calificación de graduados.

Los números entre paréntesis son coeficientes $t$.

$\mathrm{R}^{2}$ se corrige por los grados de libertad.

E1 cuadro 4 muestra algunos resultados típicos de las funciones demanda que fueron ajustadas. Cuando la variable dependiente es el porciento de graduados en la fuerza de trabajo de la empresa, el tipo de producto y la forma como se produce (electrónica en serie o producción de unidades) parece ser la única variable explicativa estadísticamente sig- 
nificativa (columna 2). Cuando las categorías educativas son agregadas y el nivel cNs y más se pone como variable dependiente, la relación mejora marginalmente, ya que la variable artificial del tamaño de la casa matriz recoge algo de la variación (columna 4). Esto quiere decir que las empresas que pertenecen a casas matrices más pequeñas tienden a emplear menos gente con calificación más alta. La productividad de la mano de obra, la productividad del capital y el producto neto parecen no relacionarse estadísticamente con el empleo de mano de obra calificada. La sustitución del producto per capita neto y la tasa de rentabilidad del capital por el capital total por persona y la productividad total de los factores, respectivamente, no cambian el resultado anterior (columna 3 ).

Se intentó después la desagregación de la variable mano de obra en cada función dentro de la empresa. En la columna 5 del cuadro 4, se usó como variable dependiente el porciento de personal de nivel cNs y superior que se empleó en investigación y desarrollo experimental. El resultado no fue mejor que en los casos anteriores. A continuación nos dirigimos a las ocupaciones particulares dentro de la empresa. La columna 6 del cuadro 4 muestra los resultados que se obtuvieron en el caso de los técnicos. La relación es un poco peor que en el caso anterior, ya que dos coeficientes pierden su significación. Finalmente, se intentó examinar la proporción de empleados con un cierto nivel educativo en una ocupación dada. La columna 7 muestra el resultado típico obtenido. EI tamaño de la casa matriz llega a ser insignificante, mientras que el coeficiente de la variable artificial "otros productos producidos en serie" gana significado estadístico.

¿Qué se ha aprendido al usar funciones demanda en vez de funciones producción? Primero, el tipo de producto y la forma en que se produce son los que guardan mejor relación con la demanda de fuerza de trabajo calificada. Segundo, la productividad total de los factores (o eficiencia económica) no tiene nada que ver con el empleo de mano de obra en proporciones dadas, es decir, no se puede rechazar la hipótesis de que empresas eficientes o ineficientes emplean fuerza de trabajo calificada en la misma proporción. Tercero, no es más fácil predecir la estructura ocupacional que la educativa. De hecho, la estructura educativa por ocupaciones es la más difícil de predecir. ${ }^{5}$

\section{INTERPRETACIÓN DE LOS RESULTADOS}

En resumen, las funciones demanda no dieron ningún resultado mejor que las funciones de producción. Independientemente del enfoque que adoptemos para nuestro problema (por el lado de la oferta o de la demanda), el resultado es el mismo: la proporción de mano de obra empleada por los distintos establecimientos no se asocia estadísticamente con prácticamente ninguna de las variables que se examinaron. ¿Qué interpretación se puede dar a este hallazgo negativo?

La teoría económica dice que si todos los establecimientos producen el mismo producto, se enfrentan a los mismos precios relativos de los factores de la producción y usan las mismas funciones producción, entonces deberán producir con idénticas relaciones de insumo de factores.

5 A juzgar por la $R^{2}$ no significativa en el cttadro 4, columna 7 . 
Considérese el caso de la gráfica 1 , donde los salarios relativos de los empleados graduados respecto de los no graduados son los indicados por la pendiente indicativa de restricción presupuestal $A B$. Si todas las empresas producen en condiciones idénticas, y los salarios relativos no varían, entonces el único punto de maximización de ganancias es $M, \mathrm{y}$ todas las empresas deberían producir con un cociente de graduados a no graduados como el que indica el rayo $O R_{0}$. Lo que se observa en este proyecto es que el rayo $O R$ varía grandemente de $O R_{1}$ a $O R_{2}$, a pesar de que los establecimientos se enfrentan a salarios relativos (línea $A B$ ) prácticamente constantes. En otras palabras, algunos establecimientos parecen producir en puntos que no son óptimos tales como $C$ y $D$. (No son óptimos en el sentido de que el mismo producto $Y$ se produce en $C$ a un costo más alto que en $M$ o, alternativamente, el punto $C$ está fuera de la restricción presupuestal original $A B$.) Sin embargo, esto no resulta verdadero en nuestra muestra en que los puntos $C$ y $D$ no se asocian a costos unitarios más altos.

Este hecho podría interpretarse como evidencia de la existencia de posibilidades de sustitución altas entre graduados y no graduados en el proceso de producción. Convendría evitar hablar acerca de las "elasticidades de sustitución" en el sentido de los libros de texto, que relaciona la proporción de graduados y no graduados con sus salarios relativos o sus productos marginales, suponiendo que son constantes todos los demás factores que operan en la decisión de la empresa para contratar mano de obra. No hemos podido calcular elasticidades a la manera del libro de texto simplemente porque estos otros factores varían mucho de un esta-

\section{Gráfica 1}

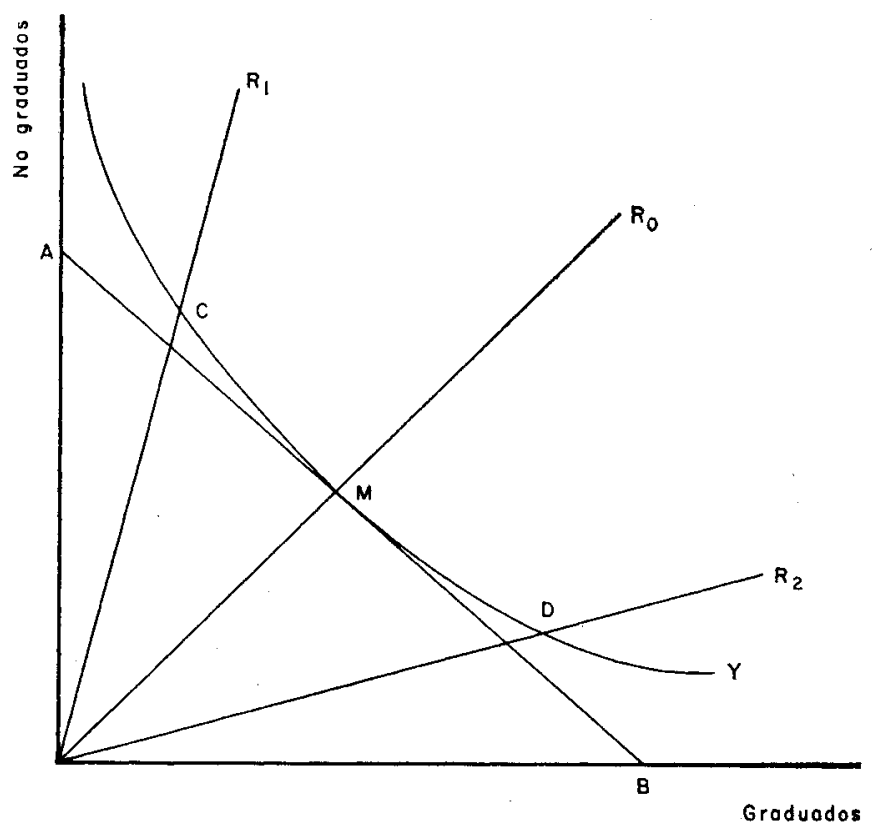


blecimiento al otro. Podría ser que los establecimientos usen técnicas de producción muy diferentes o que difieran en sus insumos administrativos o gerenciales. Lo que más nos interesa como planificadores educativos no es tanto a qué se debe esta sustitución, sino el hecho de que ésta existe. Por lo tanto, sería conveniente hablar de la "sustitución de facto" que tiene orígenes desconocidos o especulativos, más que de "elasticidades de sustitución" a la manera del libro de texto.

Recordando la forma cómo comenzó este análisis, en el caso de "sustitución fácil" no interesaría la predicción exacta de las cantidades de mano de obra. En la gráfica 1, no importa realmente si los graduados se producen en las proporciones que se indican en el renglón $O R_{0}$ en el año-meta, ya que empresas distintas podrían absorber un número grande de graduados en relación con no graduados, como lo dice el margen entre $O R_{1}$ y $O R_{2}$. Y esta absorción podría llevarse a cabo de manera eficiente debido a los factores que están ausentes en la gráfica tradicional que hemos presentado y que son muy difíciles de cuantificar en el análisis estadístico que se ha llevado a cabo (por ejemplo, funciones de producción diferentes para cada subconjunto de productos de la empresa).

Lo que en verdad importa en un caso tan flexible son los beneficios relativos al costo de "producción" de los graduados. Si las empresas pueden absorber de manera eficiente prácticamente cualquier proporción de graduados y no graduados, entonces los planificadores educativos deberán suministrar la proporción de graduados a no graduados que represente la mayor tasa de beneficio-costo social.

\section{TASAS DE RENDIMIENTO}

Esto nos lleva inevitablemente a un análisis costo-beneficio. En la muestra se ha usado información sobre las percepciones de $26000 \mathrm{em}$ pleados para estimar la tasa de rendimiento de diversas calificaciones. El cuadro 5 muestra un resumen de los resultados que se obtuvieron. ${ }^{6}$ Todas las cifras de redituabilidad son relativas a la terminación cabal de la escuela secundaria (nivel avanzado $[A$ level $]$ ). Los costos se ajustaron por las deserciones ocurridas. Se supuso que las percepciones de corte transversal de nuestra muestra crecerían a una tasa uniforme de $2 \%$, a fin de tener en cuenta el aumento de la productividad. Las diferencias

${ }^{6} \mathrm{La}$ tasa de rendimiento $\left(r_{k}\right)$ del nivel educativo $k$ se obtuvo al resolver para $r_{k}$ la ecuación siguiente

$$
\sum_{t=-s}^{o}\left(C_{k}+W_{A}\right)_{t}\left(1+r_{k}\right)^{-t}=\sum_{t=1}^{n}\left(W_{k}-W_{A}\right)\left(1+r_{k}\right)^{-t}
$$

donde $s$ corresponde al número de años de inversión en la calificación $k$,

$n$ corresponde al número de años de vida activa del graduado,

$C_{k}$ Corresponde al costo directo anual para obtener la calificación $k$,

$W_{A}$ son las percepciones del grupo de control (en este caso, graduados de la escuela secundaria a nivel $A$ ), y

$W_{k}$ corresponde a las percepciones del graduado. 
de percepciones entre niveles educativos se ajustaron hacia abajo por un factor igual a .66 (coeficiente $\alpha$ ) para tener en cuenta otros factores, además de los educativos, que influyen en las ganancias.

En el cuadro 5 aparecen dos clases de tasas de rendimiento, privadas y sociales. La tasa privada se calculó desde el punto de vista de un inversionista individual (es decir, las percepciones se consideran después de deducidos los impuestos y los costos incluyen sólo la parte que los estudiantes pagaron realmente), mientras que la tasa social se calculó desde

\section{Cuadro 5}

Gran BRetaña: TASAS de RENDIMIENTo de la inYersión EN EdUCACIÓN DE CIENCIAS E INGENIERÍA

(Porcientos)

\begin{tabular}{lcc}
\hline Nivel oducativo & Privado & Social \\
$(1)$ & $(2)$ & $(3)$ \\
\hline Diploma naoional superior (DNS) & 8.8 & 3.9 \\
Eramenes profesionales & 11.1 & 8.5 \\
Licenciaturas & 11.2 & 7.7 \\
Maestrias & 7.7 & 4.3 \\
Doctorados & 10.7 & 6.7 \\
\hline
\end{tabular}

Notas: Todos los porcientos son relativos a la terminación cabal de la escuela secundaria (nivel A).

Las tasas se ajustaron de acuerdo a la deserción, el crecimiento de la productividad $(2 \%)$ y factores adicionales a la educación que influyen en las diferencias de percepciones $(\alpha=0.66)$.

El DNS es semejante al CNS, salvo que se obtiene mediante estudios formales.

un punto de vista social (esto es, las percepciones son las habidas sin deducción de impuestos y los costos incluyen los gastos anuales totales necesarios para obtener un nivel de calificación determinado).

Estas tasas se pueden considerar desde el punto de vista de su magnitud absoluta o relativa. En términos absolutos, estas tasas no causan gran impresión. Existen probablemente otras oportunidades sociales de inversión que produzcan una tasa social de rendimiento del $3.9 \%$, esto es, más alta que la inversión en la obtención de un Diploma Nacional Superior. Sin embargo, la magnitud absoluta de las tasas de rendimiento puede ser engañosa ya que todas éstas podrían tener sesgos en dirección ascendente o descendente. Lo que resulta de más interés para los propósitos de la planeación educativa es fijarse en sus magnitudes relativas.

Por ejemplo, las tasas del cuadro 5 expresan que es más redituable, desde el punto de vista de la sociedad, producir el tipo de fuerza de trabajo que hemos llamado "profesionales" (que tenga una tasa social de rendimiento igual a $8.5 \%$ ). La siguiente oportunidad de inversión redituable serían las licenciaturas (con una tasa igual a 7.7\%), y así sucesivamente. No hay nada en el análisis de tasas de rendimiento que indique cuántos profesionales y cuántas licenciaturas se tendrían para el añometa. Estas tasas señalan únicamente la dirección del cambio del producto educativo, es decir, en este caso se alejan de los DNS y se dirigen hacia las licenciaturas y los profesionales. 


\section{CONCLUSIONES}

¿Qué hemos aprendido del proyecto de la Escuela de Economía de Londres sobre mano de obra industrial? ¿Fueron demasiado negativos los resultados en el sentido de que no fueron lo que esperaban los investigadores? ¿Hay alguna forma de volver los resultados negativos anteriores en conclusiones positivas? Esto último se podría contestar afirmativamente.

Primero, se ha aprendido que es muy difícil la estimación estadística de elasticidades de sustitución entre insumos de trabajo de distintos tipos, aun cuando se trabaje con una muestra bien diseñada y cotejada. Segundo, se ha aprendido que no es más fácil predecir la estructura ocupacional que la estructura educacional de la fuerza de trabajo. También se ha aprendido que a pesar de las dificultades para estimar las elasticidades de sustitución a la manera de los libros de texto, existe realmente una escala considerable de posibilidades de sustitución, a juzgar por los cocientes a los cuales las empresas emplean con eficiencia la fuerza de trabajo de distintos tipos. Si esta es una interpretación válida de resultados, entonces todo el análisis señala la conveniencia de usar medidas de redituabilidad en la planificación educativa, en lugar del cálculo de requerimientos de mano de obra determinados tecnológicamente.

Desde luego que el análisis de tasas de rendimiento no es panacea. He argumentado que la forma correcta de planificación educativa es una combinación de los enfoques de mano de obra y de tasa de rendimiento; además, he sugerido cómo se podría hacer esta síntesis. ${ }^{7}$ Sin embargo, baste mencionar por ahora que la evidencia del proyecto de mano de obra industrial de la Escuela de Economía de Londres muestra que el mundo real debe estar más cerca de los supuestos de quienes abogan por el método de tasas de rendimiento que de los que están implícitos en el enfoque de requerimientos de mano de obra.

7 Véase G. Psacharopoulos, Towards a Synthesis of the Manpower and the Rate of Return Approach, Higher Education Research Unit, Escuela de Economía de Londres, Programa de Estudios de Mano de Obra, Memorándum de investigación núm. 2, noviembre de 1969. 